\title{
Correlation between Topographic Progression of Geographic Atrophy and Visual Acuity Changes
}

\author{
Jong In You ${ }^{1 *}$, Do Gyun Kim²*, Seung-Young Yu ${ }^{1}$, Eung Suk Kim¹, Kiyoung Kim ${ }^{1}$ \\ ${ }^{1}$ Department of Ophthalmology, Kyung Hee University Hospital, Kyung Hee University School of Medicine, Seoul, Korea \\ ${ }^{2}$ Department of Ophthalmology, Myongji Hospital, Hanyang University College of Medicine, Goyang, Korea
}

\begin{abstract}
Purpose: To analyze topographic progression of geographic atrophy with different concentric circles centered on the fovea in correlation with decrease of visual acuity.

Methods: We retrospectively analyzed 36 eyes of 26 patients diagnosed with geographic atrophy and followed at least 1 year. One millimeter circular area at the foveal center were defined as zone 1, and doughnut shape areas from between 1 and $2 \mathrm{~mm}$ to between 5 and $6 \mathrm{~mm}$ were defined as zone 2 to 6 . Then, changes of geographic atrophy area in each zone were measured with semi-automatic software. Correlation analysis and regression analysis were performed to determine the relationship between changes in visual acuity and atrophic area in each zone.
\end{abstract}

Results: Mean age was 76.9 years and follow-up period were 3.38 years. The mean atrophic area increased from 8.09 to $16.34 \mathrm{~mm}^{2}$ and visual acuity decreased from 0.39 to 0.69 on logarithm of the minimal angle of resolution. Mean change of total geographic atrophy area was not significantly correlated with visual acuity decrease. While geographic atrophy progression within zone 1, 2, and 3 showed significant causal relationship with decrease of visual acuity (all, $p<0.05$ ).

Conclusions: In contrast to the total geographic atrophy area, progression of geographic atrophy in parafoveal area was significantly correlated with decrease of visual acuity.

Key Words: Fundus autofluorescence, Geographic atrophy, Macular degeneration

Geographic atrophy is known as the end stage of the atrophic age-related macular degeneration without choroidal neovascularization. It presents clinically as a loss of retinal pigment epithelium including overlying photoreceptors

Received: March 19, 2021 Final revision: July 12, 2021

Accepted: August 29, 2021

Corresponding Author: Kiyoung Kim, MD, PhD. Department of Ophthalmology, Kyung Hee University Hospital, 23 Kyungheedae-ro, Dongdaemun-gu, Seoul 02454, Korea. Tel: 82-2-958-8451, Fax: 82-2-966-7340, E-mail: pourma@khu.ca.kr

"These two authors contributed equally to this work. and choroidal vessels may be seen more distinctly. Geographic atrophy is estimated to cause $12 \%$ to $21 \%$ of severe blindness in age-related macular degeneration patients [1,2]. Although enlargement of atrophic lesion is the most commonly used and reproducible methods to assess disease progression, it also correlates with the decrease of visual function.

Typically, atrophic lesions appear first in the perifoveal area, sparing the foveal center, over time these lesions often expand and coalesce to include the fovea [3]. This progression is expected to cause significant visual decline, although visual acuity can decline before the fovea is affect- 
ed $[4,5]$. Patients with geographic atrophy may have scotomas, decreased reading speed, reduced contrast sensitivity and low-luminance visual activity, even when central visual acuity is preserved [6-8]. It has been reported that central vision is not completely lost and thus have relatively preserved visual acuity until the atrophic area progresses to more than $80 \%$ of the foveal center [9]. This phenomenon is related to the fact that vision may vary according to patient's ability to find minimally invaded center foveal area [10]. However, these is no conclusive result regarding how topographic progression of geographic atrophy may correlate with visual acuity changes. Therefore, the objective of this study was to find relationship between progression of geographic atrophy and visual acuity decrease, according to the involvement in different circular areas centered on the fovea.

\section{Materials and Methods}

\section{Study design}

A retrospective medical record review was performed for patients diagnosed with geographic atrophy secondary to age-related macular degeneration between February 2011 and July 2017 at our hospital. This study was performed in accordance with the Declaration of Helsinki. Institutional review board of Kyung Hee University Hospital approved the protocol (2019-05-013), and informed consent was obtained from each subject.

\section{Subjects}

Patients over 50 years of age without history of choroidal neovascularization were included in this study. Patients with at least 1 year of follow-up period were reviewed. Patients with atrophy due to high myopia (spherical equivalent $>-6$ diopters) or due to macular dystrophy were excluded. Patients with dense cataracts or corneal opacity were excluded to provide good imaging quality and analysis. Patients with previous ocular surgery including vitrectomy or pre-existing ocular disease including uveitis, severe glaucoma, and severe diabetic retinopathy were also excluded from this study.

\section{Retinal imaging procedure}

Geographic atrophy was defined as discrete areas measuring more than $500 \mathrm{~mm}$ of loss of retinal pigment epithelium with a color and thickness change relative to the sur-
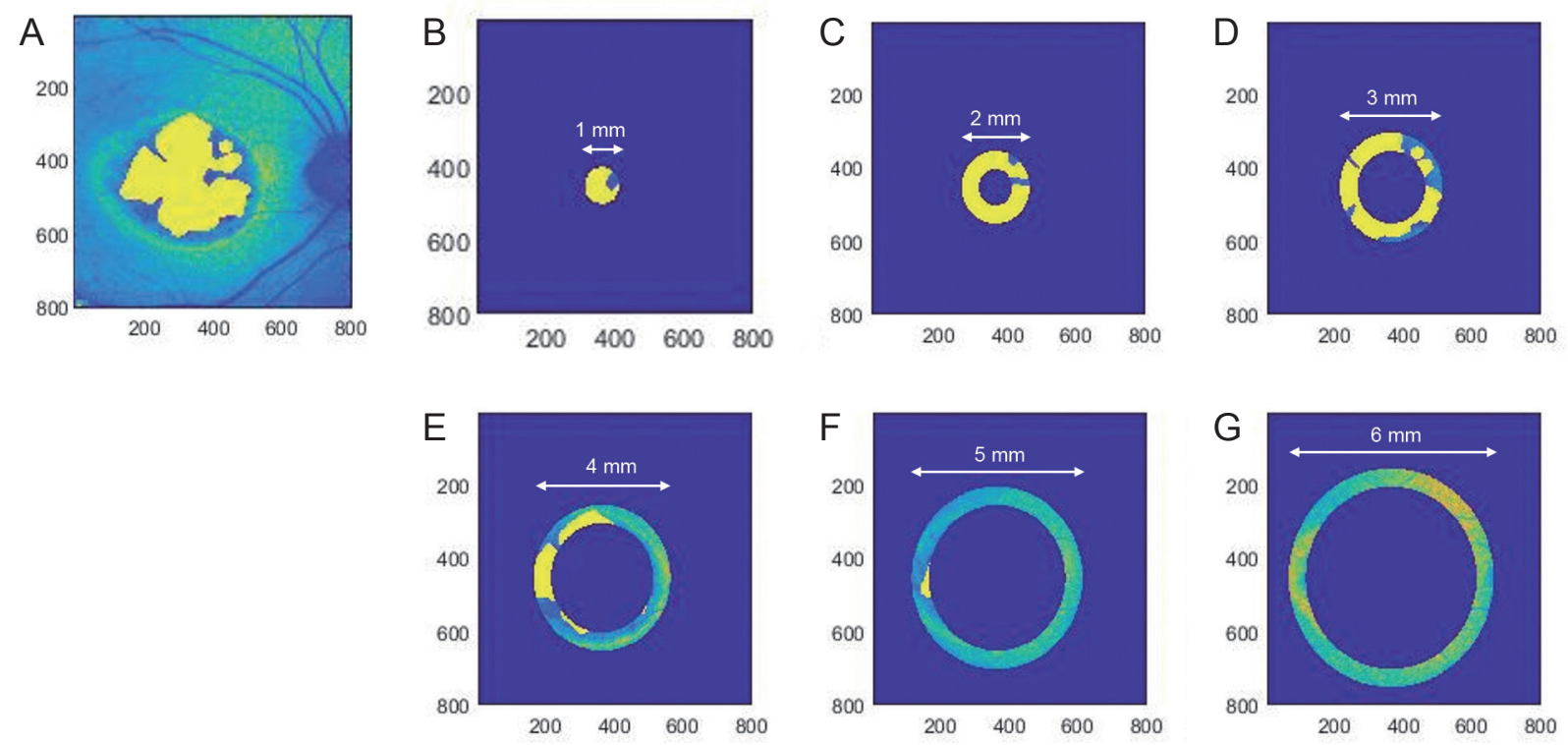

Fig. 1. Total geographic atrophic area and segmented geographic atrophy area by zone 1 to zone 6. (A) Total atrophic area, (B) zone 1, (C) zone 2, (D) zone 3, (E) zone 4, (F) zone 5, and (G) zone 6. Yellow region indicates manually measured atrophic area based on light blue autofluorescence image. Each zone was defined as doughnut shape area divided by distance of $1 \mathrm{~mm}$ from foveal center. 

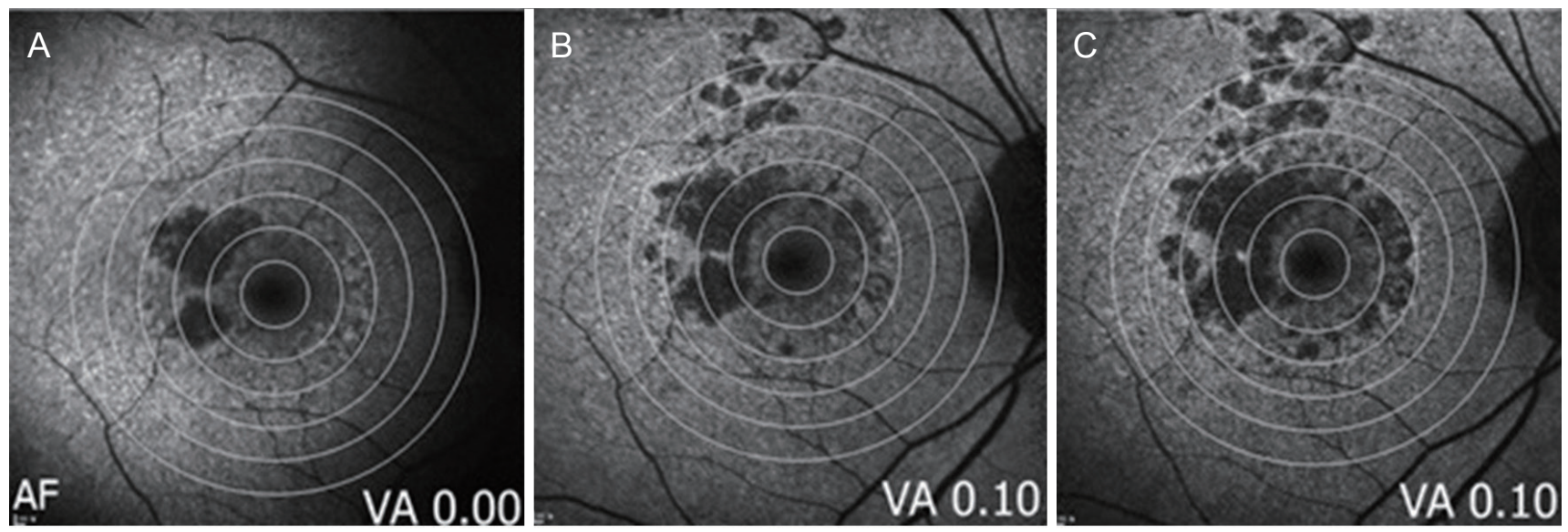

Fig. 2. A serial fundus autofluorescence image of subject with geographic atrophy. Geographic atrophy area was measured based on fundus autofluorescence image. Corresponding image was divided by six circular areas at increase of $1 \mathrm{~mm}$ from foveal center. Progression of atrophic region was observed on zone 1 through zone 6 at yearly follow-up images. (A) Baseline, (B) 1-year follow-up, and (C) 2-year follow-up. $\mathrm{AF}=$ autofluorescence; $\mathrm{VA}=$ visual acuity.

rounding retina with more prominent visualization of choroidal vessels. Atrophic area was measured based on fundus autofluorescence (FAF; $30 \times 30$ degrees and $768 \times$ 768 pixels; Spectralis HRA-OCT, Heidelberg Engineering, Heidelberg, Germany). Color fundus photography and optical coherence tomography (Spectralis HRA-OCT) were used to determine the involvement of central fovea.

\section{Image analysis and software}

Geographic atrophy area was measured manually using ImageJ ver. 1.48 (National Institutes of Health, Bethesda, MD, USA; http://imagej.nih.gov/ij/) and calculated with MATLAB ver. R2013b (The MathWorks, Natick, MA, USA). Zone 1 (fovea) was defined circle with $1 \mathrm{~mm}$ diameter centered on the fovea. Then, zone 2 to 6 were defined as doughnut-shaped area around fovea divided by concentric circles each with increase in diameter from 1 to $6 \mathrm{~mm}$ (Fig. 1A-1G). Total atrophic area and atrophic area in each zone were measured twice by one physician on two different dates and their average value was obtained.

\section{Examination protocol}

A full ophthalmic examination was performed, including best-corrected visual acuity (BCVA) using decimal chart at $4 \mathrm{~m}$, slit-lamp examination, fundus photography, FAF, and optical coherence tomography (OCT) in each visit. BCVA was converted to logarithm of the minimal angle of resolution equivalent. BCVA and change of atrophic area were then analyzed annually (Fig. 2A-2C).

\section{Statistical analysis}

Descriptive statistics were used for each area of atrophic region over time and BCVA. Relation between area and BCVA was described using mixed model analysis of covariance and generalized linear model. All statistical analyses were performed using PASW SPSS ver. 18.0 (SPSS Inc., Chicago, IL, USA). A $p$-value of $<0.05$ was considered statistically significant for all analyses.

\section{Results}

A total of 36 eyes of 26 patients with geographic atrophy secondary to age-related macular degeneration were analyzed. Mean age of patients was $76.90 \pm 7.90$ years and mean follow-up period was $3.38 \pm 1.57$ years (Supplementary Table 1,2). Among all patients, visual acuity was significantly decreased from $0.39 \pm 0.40$ to $0.69 \pm 0.64$ logarithm of the minimal angle of resolution during the follow-up period $(p<0.001)$. The atrophic area was measured twice and intraclass correlation was analyzed. Based on intraclass correlation coefficient, there was a high degree of accuracy of atrophic area on each zone (intraclass 
Table 1. Progression rate of geographic atrophy area according to the zone 1 to 6

\begin{tabular}{|c|c|c|c|}
\hline Atrophic area & Initial area $\left(\mathrm{mm}^{2}\right)$ & Rate of progression $\left(\mathrm{mm}^{2} / \mathrm{yr}\right)$ & Ratio of increase \\
\hline Total area & $8.09 \pm 0.10$ & 2.440 & 2.02 \\
\hline Zone 1 & $0.07 \pm 0.10$ & 0.011 & 1.45 \\
\hline Zone 2 & $0.23 \pm 0.18$ & 0.072 & 1.67 \\
\hline Zone 3 & $0.48 \pm 0.30$ & 0.210 & 1.84 \\
\hline Zone 4 & $0.92 \pm 0.31$ & 0.380 & 1.97 \\
\hline Zone 5 & $0.85 \pm 0.22$ & 0.510 & 1.99 \\
\hline Zone 6 & $0.95 \pm 0.45$ & 0.640 & 2.00 \\
\hline
\end{tabular}

Values are presented as mean \pm standard deviation unless otherwise indicated.

correlation coefficient $>0.93$ ) (Supplementary Table 3). Therefore, we assumed atrophic region by averaging two areas.

\section{Progression of atrophy area}

Atrophic area of each eye was compared from initial visit to the last visit. During follow-up period, atrophic area increased from 8.09 to $16.34 \mathrm{~mm}^{2}$ and progression rate of total geographic atrophy area was $2.44 \mathrm{~mm}^{2} / \mathrm{yr}$. Atrophic area of $1 \mathrm{~mm}$ from foveal center increased from 0.07 to $0.10 \mathrm{~mm}^{2}$. Rate of progression increased when distance from foveal center was increased. For example, $6 \mathrm{~mm}$ from foveal center showed the most rapid progression of atrophic area. Even if area of each region was standardized, rate of progression increased in the peripheral atrophic area (Table 1).

\section{Correlation between topographic progression of atro- phy and BCVA changes}

Causal relationship of change in atrophic area with BCVA during follow-up period was analyzed by analysis of covariance after adjusting for year, initial atrophic area, initial visual acuity, each patient, and patient's eyes. Changes in total atrophic area was not significantly correlated with decrease of BCVA $(p=0.108)$. However, zone 1 to zone 3 showed significant relationships with change of visual acuity. Zone 1 (correlation coefficient, $r=0.54$ ) and zone $2(r=0.61)$ showed more relevant causal relationship with change of vision than zone $3(r=0.44)$. Whereas zone 4 to zone 6 showed insignificant correlation with change of BCVA (Table 2).

Causal relationship between change of atrophic area and
Table 2. Causal relationship of change of atrophic area with visual acuity according to the zone 1 to zone 6

\begin{tabular}{lcc}
\hline Atrophic area & Correlation coefficient (r) & $p$-value \\
\hline Total area & 0.10 & 0.108 \\
Zone 1 & 0.54 & $0.021^{*}$ \\
Zone 2 & 0.61 & $0.010^{*}$ \\
Zone 3 & 0.44 & $0.032^{*}$ \\
Zone 4 & 0.35 & 0.099 \\
Zone 5 & 0.22 & 0.167 \\
Zone 6 & 0.18 & 0.103 \\
\hline
\end{tabular}

Analyzed by analysis of covariance after adjusting for year, initial atrophic area, initial visual acuity, each patient, and patient's eyes.

${ }^{*}$ Statistically significant value $p<0.05$.

BCVA was analyzed using generalized linear model. Among divided zones, zone 2 to zone 4 showed relationship with change of visual acuity. Zone 2 had the most relevant relationship with visual change $(\mathrm{r}=0.73, p<0.001)$ (Supplementary Table 4).

\section{Discussion}

Geographic atrophy is an irreversible, progressive vision-threatening disorder, and there has been no satisfactory method for rating disease severity affecting individual's visual function. In the most recent Chroma and Spectri study, correlation coefficients between geographic atrophy lesion size and visual function showed moderate to poor associations [11]. Therefore, we evaluated progression of geographic atrophy on dividing area according to the dif- 
ferent size of concentric circles from fovea and attempted to find their correlations with the decrease of visual acuity.

Overall, progression rates of geographic atrophy were reported in the literature for study populations range from 0.534 to $2.64 \mathrm{~mm}^{2} / \mathrm{yr}[12,13]$. Our research showed that the progression rate of geographic atrophy was $2.44 \mathrm{~mm}^{2} / \mathrm{yr}$, which was comparable with that of previous studies. In addition, risk factors for faster rates of geographic atrophy lesion enlargement was identified as larger baseline lesion size and extrafoveal location, in Chroma and Spectri study [11]. Similarly, progression of geographic atrophy was observed be faster with increasing distance from the foveal center in the present study.

In this study, we focused on causal relationship between progression of geographic atrophy in specific area and change of vision. Consistent with previous reports, change in total atrophic area was not significantly was correlated with visual changes. However, after adjusting for initial factors including initial area and initial visual acuity, significant relationship between geographic atrophy progression and visual acuity was observed in zone 1, 2, and 3 defined as parafoveal area. To the best of our knowledge, this is the first long-term study aimed to evaluate growth rate of geographic atrophy in Korean population. Also, we firstly reported to reveal geographic atrophy progression in divided concentric circular areas associated with change of vision.

Photoreceptor layer on fovea is consisted of entirely cone cell. It is also known as the most important area of maximum visual acuity. In that sense, progression of geographic atrophy in zone 1 ( $1 \mathrm{~mm}$ from foveal center) had a strong effect on decrease of visual acuity. Interestingly, zone 2 (1 to $2 \mathrm{~mm}$ from foveal center) had the strongest correlation with visual acuity. The effect of progression in atrophic area on visual acuity gradually decreased from parafoveal to perifoveal area. This phenomenon can be related to characteristic of autofluorescence image to detect lipofuscin. The maximum intensity on parafoveal region decreases towards the retina periphery on autofluorescence image [14]. Lower intensity on foveal center is due to foveal luteal pigment absorbing blue light [15]. Moreover, higher melanin granule and lower lipofucin granule in central retinal pigment epithelium are associated with lower intensity [16]. Therefore, single autofluorescence image is not suitable to evaluate degeneration on foveal center. On the other hand, degeneration in the parafoveal region can be easily detected due to higher intensity on autofluorescence. Although we carefully measured atrophic area of foveal center by using multimodal image including fundus photography and OCT, we could not exactly measure damage of specific cell type by using these images. Consequently, the inconsistency of clinical image of atrophic area over degeneration on cellular level can be one reason for explaining the importance of parafoveal area over foveal center.

It is well-known that parafoveal region can support central vision and visual acuity. Rods are known to produce diffusible substance essential for cone survival [17,18]. If rods are mostly present in the parafoveal area, parafoveal region can affect visual acuity. Geographic atrophy lesions may grow into foveal surrounding horseshoe or ring shapes [5]. It was hypothesized that preferential foveal sparing reflects the relatively lower susceptibility of cone versus rod photoreceptors to cell death $[19,20]$. Recently with the development of OCT angiography, degeneration areas of geographic atrophy are found not only on photoreceptor and retinal pigment epithelium, but also on choriocapillaris, the innermost layer of choroid [21]. Choriocapillaris is known to support retinal pigment epithelium and photoreceptor layer. In that case, microvascular degeneration of geographic atrophy over parafoveal area can induce decrease of visual acuity over time. A recent study has revealed the association of parafoveal area and visual acuity [22,23]. Sandberg et al. [17] have reported that visual acuity is not only related to foveal area, but also related to parafoveal retinal thickness in retinitis pigmentosa. Another study has revealed the correlation of change in microvascular structures with visual acuity in retinal vein occlusion by using OCT angiography [23]. In this report, superficial foveal avascular zone and parafoveal vascular density are correlated with visual acuity in retinal vein occlusion. In that sense, further study will be needed to find the relationship of microvascular degeneration and atrophic area correlating with visual change.

This study has some limitations. First, the number of patients and eyes involved was relatively small and follow-up period was not consistent. Secondly, due to the retrospective nature of this study, only BCVA was explored to estimate visual deficit in geographic atrophy. It is recognized that BCVA often underrepresents functional deficits, especially in those patients with foveal sparing-lesions [24]. Other studies have evaluated correlations between geographic atrophic lesion area and alternative measures of vi- 
sual function, such as, low-luminance visual acuity, microperimetry, and reading speed. Thirdly, geographic atrophy size was evaluated using FAF imaging, which visualizes decreased autofluorescence. Loss of the photoreceptor components or choriocapillaris may not show as abnormalities on FAF, and thus the use of FAF-detected geographic atrophy area may not appropriately reflect the true extent of cell death or dysfunction and thus may not correlate as well as may be expected with decline in visual function $[25,26]$. Also, due to fovea absorbing blue light autofluorescence, measuring foveal involvement in the presence of geographic atrophy by short-wavelength FAF alone may be challenging [3]. Therefore, we used other imaging modalities including color fundus photo and OCT in confirming lesion boundaries. However, the degree of precision wtihin measured atrophic area around the fovea can affect the validity of progression of zone 1 .

Currently, many factors potentially prognostic for geographic atrophy progression have been identified, yet imaging parameter for rating an individual's severity is limited, particularly those affecting visual function. For example, although lesion size is known to be important, the degree of foveal involvement and impact of parafoveal lesions are critical to determining geographic atrophy lesions to visual acuity. Therefore, our results suggested that geographic atrophy progression including parafoveal area, especially within $3 \mathrm{~mm}$ from foveal center, had significant correlation with visual acuity, in contrast to the progression of total atrophic area. Geographic atrophy progression within parafoveal area should carefully be monitored for decrease of vision. Further study would be warranted to understand how this topographic involvement of geographic atrophy exactly interact and relate to the central visual function.

\section{Conflict of Interest}

No potential conflict of interest relevant to this article was reported.

\section{Supplementary Materials}

Supplementary MaterialsSupplementary Tables are available from: https://doi.org/10.3341/kjo.2021.0037.

\section{References}

1. Sunness JS. The natural history of geographic atrophy, the advanced atrophic form of age-related macular degeneration. Mol Vis 1999;5:25.

2. Gao H, Hollyfield JG. Aging of the human retina. Differential loss of neurons and retinal pigment epithelial cells. Invest Ophthalmol Vis Sci 1992;33:1-17.

3. Lindner M, Boker A, Mauschitz MM, et al. Directional kinetics of geographic atrophy progression in age-related macular degeneration with foveal sparing. Ophthalmology 2015;122:1356-65.

4. Fleckenstein M, Mitchell P, Freund KB, et al. The progression of geographic atrophy secondary to age-related macular degeneration. Ophthalmology 2018;125:369-90.

5. Sunness JS, Gonzalez-Baron J, Applegate CA, et al. Enlargement of atrophy and visual acuity loss in the geographic atrophy form of age-related macular degeneration. Ophthalmology 1999;106:1768-79.

6. Sunness JS, Rubin GS, Broman A, et al. Low luminance visual dysfunction as a predictor of subsequent visual acuity loss from geographic atrophy in age-related macular degeneration. Ophthalmology 2008;115:1480-8.

7. Sunness JS, Rubin GS, Applegate CA, et al. Visual function abnormalities and prognosis in eyes with age-related geographic atrophy of the macula and good visual acuity. Ophthalmology 1997;104:1677-91.

8. Congdon N, O'Colmain B, Klaver CC, et al. Causes and prevalence of visual impairment among adults in the United States. Arch Ophthalmol 2004;122:477-85.

9. Sarks JP, Sarks SH, Killingsworth MC. Evolution of geographic atrophy of the retinal pigment epithelium. Eye (Lond) 1988;2(Pt 5):552-77.

10. Sayegh RG, Sacu S, Dunavolgyi R, et al. Geographic atrophy and foveal-sparing changes related to visual acuity in patients with dry age-related macular degeneration over time. Am J Ophthalmol 2017;179:118-28.

11. Heier JS, Pieramici D, Chakravarthy U, et al. Visual function decline resulting from geographic atrophy: results from the Chroma and Spectri phase 3 trials. Ophthalmol Retina 2020;4:673-88.

12. Batioglu F, Gedik Oguz Y, Demirel S, Ozmert E. Geographic atrophy progression in eyes with age-related macular degeneration: role of fundus autofluorescence patterns, fellow eye and baseline atrophy area. Ophthalmic Res 2014;52:53-9. 
13. Sunness JS, Margalit E, Srikumaran D, et al. The longterm natural history of geographic atrophy from age-related macular degeneration: enlargement of atrophy and implications for interventional clinical trials. Ophthalmology 2007;114:271-7.

14. Ach T, Huisingh C, McGwin G Jr, et al. Quantitative autofluorescence and cell density maps of the human retinal pigment epithelium. Invest Ophthalmol Vis Sci 2014;55:4832-41.

15. Hicks D, Sahel J. The implications of rod-dependent cone survival for basic and clinical research. Invest Ophthalmol Vis Sci 1999;40:3071-4.

16. Kvanta A, Casselholm de Salles M, Amren U, Bartuma H. Optical coherence tomography angiography of the foveal microvasculature in geographic atrophy. Retina 2017;37:93642.

17. Sandberg MA, Brockhurst RJ, Gaudio AR, Berson EL. Visual acuity is related to parafoveal retinal thickness in patients with retinitis pigmentosa and macular cysts. Invest Ophthalmol Vis Sci 2008;49:4568-72.

18. Kang JW, Yoo R, Jo YH, Kim HC. Correlation of microvascular structures on optical coherence tomography angiography with visual acuity in retinal vein occlusion. Retina 2017;37:1700-9.

19. Curcio CA, Medeiros NE, Millican CL. Photoreceptor loss in age-related macular degeneration. Invest Ophthalmol Vis Sci 1996;37:1236-49.
20. Owsley C, McGwin G Jr, Jackson GR, et al. Cone- and rod-mediated dark adaptation impairment in age-related maculopathy. Ophthalmology 2007;114:1728-35.

21. Holz FG, Bindewald-Wittich A, Fleckenstein M, et al. Progression of geographic atrophy and impact of fundus autofluorescence patterns in age-related macular degeneration. Am J Ophthalmol 2007;143:463-72.

22. Choudhry N, Giani A, Miller JW. Fundus autofluorescence in geographic atrophy: a review. Semin Ophthalmol 2010;25:206-13.

23. Delori FC, Dorey CK, Staurenghi G, et al. In vivo fluorescence of the ocular fundus exhibits retinal pigment epithelium lipofuscin characteristics. Invest Ophthalmol Vis Sci 1995;36:718-29.

24. Sunness JS, Rubin GS, Zuckerbrod A, Applegate CA. Foveal-sparing scotomas in advanced dry age-related macular degeneration. J Vis Impair Blind 2008;102:600-10.

25. Sayegh RG, Simader C, Scheschy U, et al. A systematic comparison of spectral-domain optical coherence tomography and fundus autofluorescence in patients with geographic atrophy. Ophthalmology 2011;118:1844-51.

26. Panorgias A, Zawadzki RJ, Capps AG, et al. Multimodal assessment of microscopic morphology and retinal function in patients with geographic atrophy. Invest Ophthalmol Vis Sci 2013;54:4372-84. 
Supplementary Table 1. Baseline characteristics

\begin{tabular}{|c|c|c|c|c|c|c|c|}
\hline No. & Patient & $\begin{array}{l}\text { Visual acuity } \\
\text { (logMAR) }\end{array}$ & $\begin{array}{c}\text { Configuration } \\
{[4]}\end{array}$ & Inner zone & $\begin{array}{l}\text { Inner zone } \\
\text { area }\end{array}$ & $\begin{array}{l}\text { Progression to inner } \\
\text { zone }\end{array}$ & Status \\
\hline 1 & Patient 1 & 0.7 & Unifocal & 1 & 0.19 & - & Phakic \\
\hline 2 & Patient 2 & 0.3 & Unifocal & 3 & 0.02 & Stationary & Pseudo \\
\hline 3 & Patient 2 & 0.3 & Multifocal & 6 & 0.08 & Stationary & Pseudo \\
\hline 4 & Patient 3 & 0.2 & Multifocal & 1 & 0.02 & - & Phakic \\
\hline 5 & Patient 3 & 0.2 & Multifocal & 2 & 0.08 & Stationary & Phakic \\
\hline 6 & Patient 4 & 1.3 & Multifocal & 1 & 0.6 & - & Pseudo \\
\hline 7 & Patient 4 & 0.5 & Multifocal & 1 & 0.44 & - & Pseudo \\
\hline 8 & Patient 5 & 0.3 & Multifocal & 1 & 0.74 & - & Phakic \\
\hline 9 & Patient 6 & 0.05 & Multifocal & 2 & 0.12 & 2 Year to zone 1 & Pseudo \\
\hline 10 & Patient 6 & 0.05 & Multifocal & 2 & 0.06 & 2 Year to zone 1 & Phakic \\
\hline 11 & Patient 7 & 0.4 & Multifocal & 1 & 0.91 & - & Phakic \\
\hline 12 & Patient 7 & 0.3 & Multifocal & 1 & 0.65 & - & Phakic \\
\hline 13 & Patient 8 & 0.5 & Unifocal & 1 & 0.5 & - & Phakic \\
\hline 14 & Patient 8 & 0.8 & Unifocal & 1 & 0.64 & - & Phakic \\
\hline 15 & Patient 9 & 0 & Multifocal & 3 & 1.03 & 1 Year to zone 2 & Phakic \\
\hline 16 & Patient 9 & 0.15 & Multifocal & 2 & 0.09 & Stationary & Phakic \\
\hline 17 & Patient 10 & 0.05 & Unifocal & 2 & 0.01 & 1 Year to zone 1 & Phakic \\
\hline 18 & Patient 10 & 0.05 & Multifocal & 3 & 0.19 & 1 Year to zone 2 & Phakic \\
\hline 19 & Patient 11 & 0.15 & Unifocal & 1 & 0.08 & - & Phakic \\
\hline 20 & Patient 12 & 0.15 & Multifocal & 3 & 0.02 & 1 Year to zone 2 & Pseudo \\
\hline 21 & Patient 13 & 0.4 & Multifocal & 2 & 0.07 & Stationary & Phakic \\
\hline 22 & Patient 14 & 1.0 & Multifocal & 1 & 0.78 & - & Phakic \\
\hline 23 & Patient 15 & 0 & Multifocal & 2 & 0.34 & Stationary & Phakic \\
\hline 24 & Patient 16 & 0.3 & Unifocal & 2 & 0.15 & 3 Year to zone 1 & Phakic \\
\hline 25 & Patient 17 & 1.6 & Multifocal & 1 & 0.68 & - & Phakic \\
\hline 26 & Patient 18 & 0.3 & Multifocal & 1 & 0.78 & - & Phakic \\
\hline 27 & Patient 19 & 0.1 & Multifocal & 4 & 0.08 & Stationary & Phakic \\
\hline 28 & Patient 19 & 0.1 & Multifocal & 4 & 0.08 & Stationary & Phakic \\
\hline 29 & Patient 20 & 0.9 & Unifocal & 1 & 1.05 & - & Phakic \\
\hline 30 & Patient 20 & 0.9 & Unifocal & 1 & 1.2 & - & Phakic \\
\hline 31 & Patient 21 & 0.3 & Multifocal & 2 & 0.18 & 1 Year to zone 1 & Phakic \\
\hline 32 & Patient 22 & 0.5 & Unifocal & 3 & 0.6 & Stationary & Phakic \\
\hline 33 & Patient 23 & 0.4 & Unifocal & 1 & 0.09 & - & Phakic \\
\hline 34 & Patient 24 & 0.4 & Multifocal & 1 & 0.05 & - & Phakic \\
\hline 35 & Patient 25 & 0.2 & Unifocal & 5 & 0.68 & 2 Year to zone 4 & Phakic \\
\hline 36 & Patient 26 & 0.3 & Unifocal & 3 & 0.07 & Stationary & Phakic \\
\hline
\end{tabular}

$\log \mathrm{MAR}=\operatorname{logarithm}$ of the minimal angle of resolution. 
Korean J Ophthalmol Vol.35, No.6, 2021

Supplementary Table 2. The number of eyes divided by follow-up period

\begin{tabular}{lc}
\hline Mean follow-up period (yr) & No. of eyes $(\%)$ \\
\hline 1 & $5(13.89)$ \\
2 & $6(16.67)$ \\
3 & $8(22.22)$ \\
4 & $9(25.00)$ \\
5 & $3(8.33)$ \\
6 & $5(13.89)$ \\
Total & $36(100)$ \\
\hline
\end{tabular}


Supplementary 3. The degree of accuracy of measured atrophic area was analyzed using intraclass correlation

\begin{tabular}{lcc}
\hline Variable & Intraclass correlation coefficient $(95 \%$ confidence interval) & $p$-value \\
\hline Total area trial 1 \& & $0.98(0.97-0.99)$ & $<0.001$ \\
Zone 1 & $1.00(1.00-1.00)$ & $<0.001$ \\
Zone 2 & $0.99(0.98-0.99)$ & $<0.001$ \\
Zone 3 & $0.98(0.97-0.99)$ & $<0.001$ \\
Zone 4 & $0.98(0.96-0.98)$ & $<0.001$ \\
Zone 5 & $0.98(0.97-0.99)$ & $<0.001$ \\
Zone 6 & $0.93(0.90-0.95)$ & $<0.001$ \\
\hline
\end{tabular}


Korean J Ophthalmol Vol.35, No.6, 2021

Supplementary 4. Relationship between decease of best corrected visual acuity and change of atrophic area was analyzed using generalized linear model

\begin{tabular}{lcc}
\hline Atrophic area & Coefficient (r) & $p$-value \\
\hline Total area & 0.51 & 0.062 \\
Zone 1 & 0.71 & $0.028^{*}$ \\
Zone 2 & 0.73 & $0.002^{*}$ \\
Zone 3 & 0.71 & $0.002^{*}$ \\
Zone 4 & 0.59 & $0.008^{*}$ \\
Zone 5 & 0.50 & 0.610 \\
Zone 6 & 0.41 & 0.058 \\
\hline
\end{tabular}

*Statistically significant value. 